\title{
Research on LDPC Coding for Free Space Optical Communication with Modified Bessel-Gaussian Beams Based on K-distribution
}

\author{
Jin Wang ${ }^{a}$, Fujiang Zeng, Qingqing Liang and Xiaofeng Zhang \\ China University of Geosciences, School of Mechanical Engineering and Electronic Information, Wuhan, China
}

\begin{abstract}
We investigate the performance of low-density parity-check (LDPC) coding for free space optical communication (FSO) with modified Bessel-Gaussian (MBG) beams based on $\mathrm{K}$-distribution. Based on an extended Huygens-Fresnel principle, the receiver plane intensity is formulated and then we analyze the propagation characteristics of MBG beams traveling in a turbulent atmosphere. Numerical calculations show that the interaction of the transmission distance, refractive index structure constant, and wavelength will affect the imaginary term in the beam, allowing the beam to exhibit the properties of the MBG beams. To further improve the communication quality of the FSO system, we introduce a fast Quasi-Cyclic (QC) Low-Density Parity-Check (LDPC) code. Simulation results show that under weak and strong turbulence conditions, the performance of the QC-LDPC code becomes better and better as the code rate increases, and the bit error rate (BER) keeps decreasing as the code rate increases.
\end{abstract}

\section{Introduction}

Free Space Optical communication (FSO) is an optical communication technology that uses laser as an information carrier to propagate in free space to wirelessly transmit data for telecommunications or computer networking. FSO is characterized by ease of deployment, license-free, high bit rate, and immunity to electromagnetic interference, has attracted extensive research interests, and FSO is an important development direction of next-generation wireless communications [1,2]. Nevertheless, FSO are transmitted over atmospheric random channels, and atmospheric turbulence has a dispersion and absorption effect on the laser beam, this results in a weakened optical intensity and scintillation, eventually increased the bit error rate. In order to decrease the impact of atmospheric turbulence, we introduce the modified Bessel-Gaussian beams and the low-density parity-check (LDPC) code.

The modified Bessel-Gaussian beams has limited energy and is a physically achievable non-diffracting beams [3]. It can not only maintain its horizontal light intensity distribution within a limited propagation range, and it also has self-recovery performance when it is disturbed. A recent investigation indicates that Bessel beams possess a comparatively low scintillation index in all atmospheric regions when appropriate coherence parameters are selected.

In information theory, the low-density parity-check (LDPC) code is a linear error correcting code, a method of transmitting a message over a noisy transmission channel [4]. An LDPC is constructed using a sparse bipartite graph. LDPC codes are capacity-approaching codes, which means that

\footnotetext{
${ }^{a}$ Corresponding author : wangjin@cug.edu.cn
} 
practical constructions exist that allow the noise threshold to be set very close (or even arbitrarily close on the binary erasure channel) to the theoretical maximum (the Shannon limit) for a symmetric memoryless channel.

In this paper, we discuss the effects of modified Bessel-Gaussian beams' parameters on FSO communication system. On the other hand, the performance of LDPC code for atmospheric turbulence channels are analyzed in this paper.

The remainder of this paper is organized as follows. In Section II, the modified Bessel-gaussian beams and the LDPC code is described in detail. Section III presents the numerical calculations and analysis. Finally, Section IV gives the conclusion of this paper.

\section{Theoretical formulations}

\subsection{Modified Bessel-gaussian beams}

The modified Bessel-Gaussian beams is an electromagnetic wave beams whose transverse electric field and irradiance distribution approximately satisfy the modified Bessel-Gaussian function. Modified Bessel-Gaussian beams is improved by Bessel beams and Gaussian beams. On a source plane whose radial and azimuthal coordinates are $s$ and $\varphi_{s}$, the field of modified Bessel-Gaussian beams is given by [5]

$$
u_{s}\left(s, \varphi_{s}\right)=I_{n}\left(a_{B} s\right) \exp \left(-k \alpha s^{2}\right) \exp \left(-j n \varphi_{s}\right)
$$

Where the modified Bessel functions having order $n$, width parameter $a_{B}, k=2 \pi / \lambda$ is the wave number with $\lambda$ being the wavelength, $\alpha=1 /\left(k \alpha^{2}\right)+j / 2 F_{s}$, where $\alpha_{s}$ and $F_{s}$, respectively, refer to radial Gaussian source size and focusing parameter, $j=\sqrt{-1}$.

Therefore, the formula for receiving light intensity at the receiving surface affected by the Bessel-Gaussian beam turbulence by the extended Huygens-Fresnel principle is [6]

$$
\begin{aligned}
& \left\langle I_{r}\left(r, \varphi_{r}\right)\right\rangle \\
& =\left(\frac{k}{2 \pi L}\right)^{2} \int_{0}^{\infty} \int_{0}^{2 \pi} \int_{0}^{\infty} \int_{0}^{2 \pi} d s_{1} d \varphi_{1 s} d s_{2} d \varphi_{2 s} u_{s}\left(s_{1}, \varphi_{1 s}\right) u_{s}^{*}\left(s_{2}, \varphi_{2 s}\right) s_{1} s_{2} \\
& \quad \times \exp \left\{\frac{j k}{2 L}\left[s_{1}^{2}-s_{2}^{2}-2 r s_{1} \cos \left(\varphi_{r}-\varphi_{1 s}\right)+2 r s_{2} \cos \left(\varphi_{r}-\varphi_{2 s}\right)\right]\right\} \\
& \quad \times \exp \left\{\left[-s_{1}^{2}-s_{2}^{2}+2 s_{1} s_{2} \cos \left(\varphi_{1 s}-\varphi_{2 s}\right)\right] / \rho_{0}^{2}\right\}
\end{aligned}
$$

where $*$ stands for the complex conjugate, $\rho_{0}$ measures the coherence length of a spherical wave propagating in the turbulent medium and will be given as $\rho_{0}=\left(0.545 C_{n}^{2} k^{2} L\right)^{-3 / 5}, C_{n}^{2}$ is the refractive index structure constant indicating the turbulence strength.

When the beam diameter is larger than the turbulence, the intensity scintillation will be appeared. Under weak turbulent, the intensity scintillation is given by $\sigma_{\ln I}^{2}=0.496 k^{7 / 6} L^{11 / 6} C_{n}^{2}$, and the strong turbulence is $\sigma_{\ln I}^{2}=1.23 k^{7 / 6} L^{11 / 6} C_{n}^{2}[7]$.

\subsection{Low-density parity-check (LDPC) code}

In LDPC code, the minimum Hamming distance will increase linearly with the increase of the codeword length. Hence, the posteriori probability iterative decoding has the characteristic that the bit error rate decreases as the codeword length increases and Iterative decoding algorithm of LDPC code 
is close to the performance of Shannon limit [8,9]. LDPC codes can use random structure or algebraic method. The performance of random constructed LDPC code always is not ideal when using short code word length and the higher error flat layer would be easier to appear. The randomness of the structure would make it complex to decode. The algebraic structure of LDPC codes only exist in a specific code length and their bit rate at most of the time, have poor adaptability. In line with this, we applied a kind of LDPC code for fast encoding know as Quasi-cyclic LDPC Code (QC-LDPC). The QC-LDPC construction method is given as follows:

a) The rate is given as $R=(N-M) / N$, where $\mathrm{N}$ is the codeword length, $\mathrm{M}$ is information bit. It is important to note that the Matrix dimension is $\mathrm{q}=\mathrm{N} / 6$, with prime number $\mathrm{a}$ and $\mathrm{b}$ is smaller than q.

b) The parity-check matrix $\mathrm{H}$ is constructed by sub-matrix: $\mathrm{H}=\left[\begin{array}{ll}A & B\end{array}\right]$

$$
A=\left[\begin{array}{ccc}
I_{0} & I_{a} & I_{a^{2}} \\
0 & I_{0} & I_{a^{2} b} \\
I_{b^{2}} & 0 & I_{0}
\end{array}\right] B=\left[\begin{array}{ccc}
I_{a^{3}} & I_{a^{4}} & I_{a^{5}} \\
I_{a^{3} b} & I_{a^{4} b} & I_{a^{5} b} \\
I_{a^{3} b^{2}} & I_{a^{4} b^{2}} & I_{a^{5} b^{2}}
\end{array}\right]
$$

Here, $I_{x}$ consist of non-0 elements in A and B where $I_{x}=q \times q$ unit cyclic matrix which iterates each row of the identity matrix by $\mathrm{x}$ bits.

c) If $\mathrm{A}$ is singular, return to step 1 to re-select $\mathrm{N}$. If $\mathrm{A}$ is non-singular, we can obtain the generator matrix $\mathrm{G}$ and a code named $c_{n}$ that length is $\mathrm{N}$ by the characteristics of Parity Check Matrix.

$$
G=\left[\begin{array}{ll}
A^{-1} \cdot \mathrm{B} & I_{N-M}
\end{array}\right] ; c_{n}=u_{n} \cdot G
$$

In the equation, $u_{n}=\left[\begin{array}{llll}u_{n, 0} & u_{n, 1} & \cdots & U_{n, \mathrm{M}-1}\end{array}\right]$ is a randomly selected information bit vector. The decoding algorithm according to the method in the literature [10].

\section{Numerical calculations and analysis}

In this section, we calculate the propagation properties of the modified Bessel-Gaussian beams in atmospheric turbulence and analyze the influence of main parameters on the received optical intensity. At the end, we analyzed the performance of LDPC code in FSO.

\subsection{Optical intensity distribution}

\subsubsection{Source intensity}

Initially we start with source intensity graphs. Fig 1 shows source plane intensity of modified Bessel-Gaussian beams at various orders. From fig 1, it is seen that higher order modified Bessel-Gaussian beams, that is $n>0$, will have well-like shape whose circumference is enlarged with increasing order. From fig 2, it shows that raising the Bessel width parameter $a_{B}$ will have a similar role.

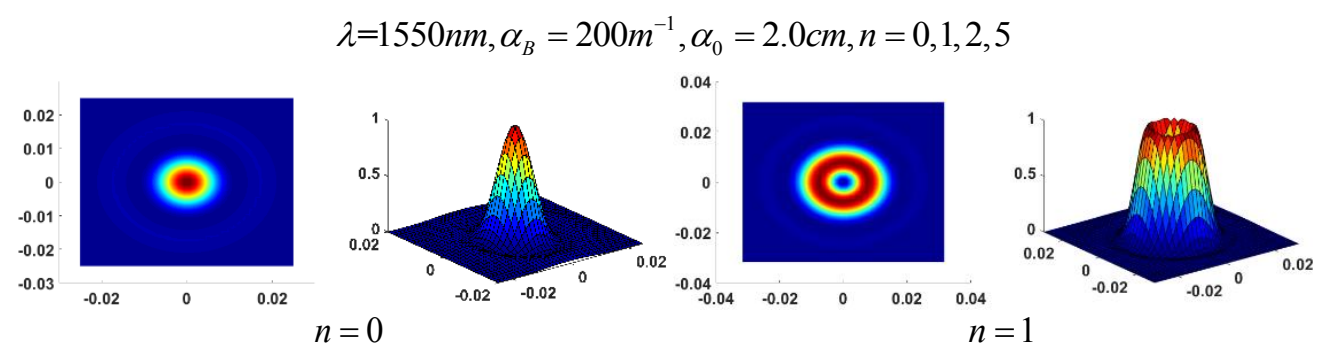



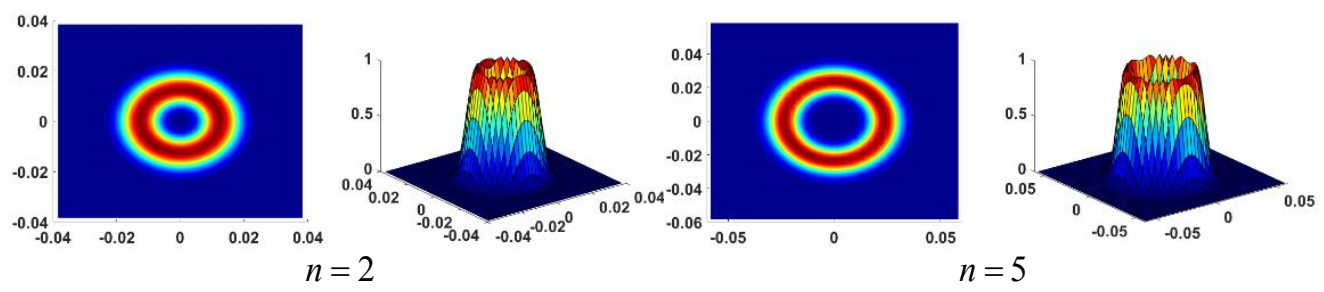

Figure 1. Source plane intensity pictures of modified Bessel-Gaussian beams at various orders.

$$
\lambda=1550, n=1, \alpha_{0}=2.0 \mathrm{~cm}, a_{B}=500,400,200,100 \mathrm{~m}^{-1}
$$
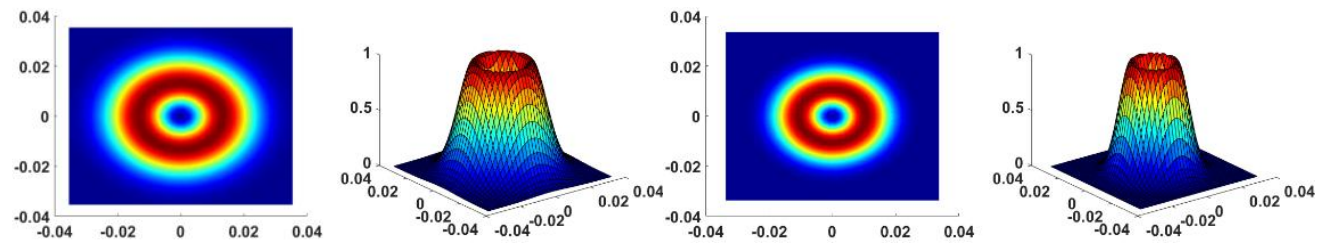

$a_{B}=500 m^{-1}$

$a_{B}=400 m^{-1}$

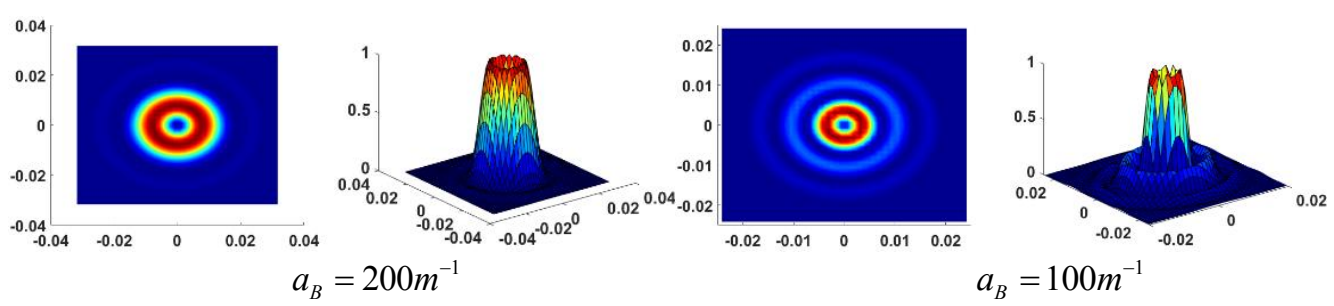

Figure 2. Source plane intensity pictures of modified Bessel-Gaussian beams at various width parameter.

\subsubsection{Ideal receiver intensity}

Now, receiver intensity graphs shall be offered. It is main describes the ideal receiver intensity in this section. Figure 3 displays the progress of modified Bessel-Gaussian beams along the propagation axis.

$$
\lambda=1550, n=0, a_{B}=250 \mathrm{~m}^{-1}, C_{n}^{2}=1 \times 10^{-15} \mathrm{~m}^{-2 / 3}
$$
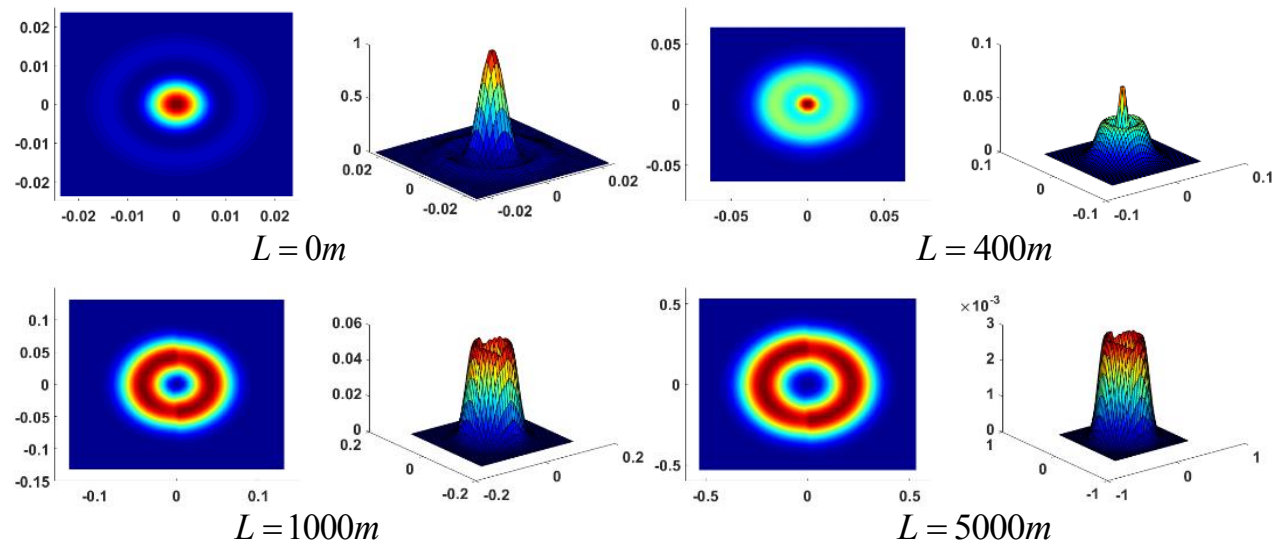

Figure 3. Ideal receiver intensity pictures of modified Bessel-Gaussian beams at various propagation lengths.

It is possible to detect from Fig 3 that, the modified Bessel-Gaussian beams will too lead to Gaussian beams after propagation in turbulent atmosphere.

Fig 4 exhibits ideal receiver intensity of modified Bessel-Gaussian beams at various $C_{n}^{2}$ values. Looking at Fig 4, it is possible to deduce that increasing the turbulence strength simply accelerates the process toward the eventual beam shape, thus a Gaussian beam is attained earlier. 

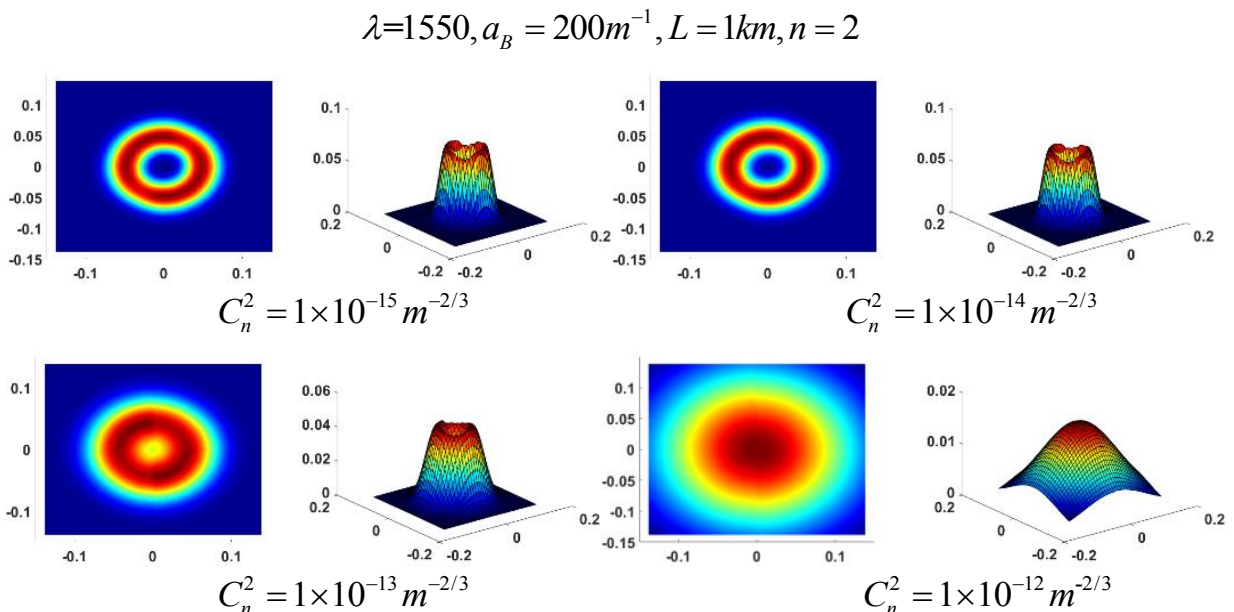

$$
C_{n}^{2}=1 \times 10^{-13} \mathrm{~m}^{-2 / 3} \quad C_{n}^{2}=1 \times 10^{-12} \mathrm{~m}^{-2 / 3}
$$

Figure 4. Ideal receiver intensity pictures of modified Bessel-Gaussian beams at various $C_{n}^{2}$ values.

$$
a_{B}=200 \mathrm{~m}^{-1}, L=1 \mathrm{~km}, n=2, C_{n}^{2}=1 \times 10^{-15} \mathrm{~m}^{-2 / 3}
$$
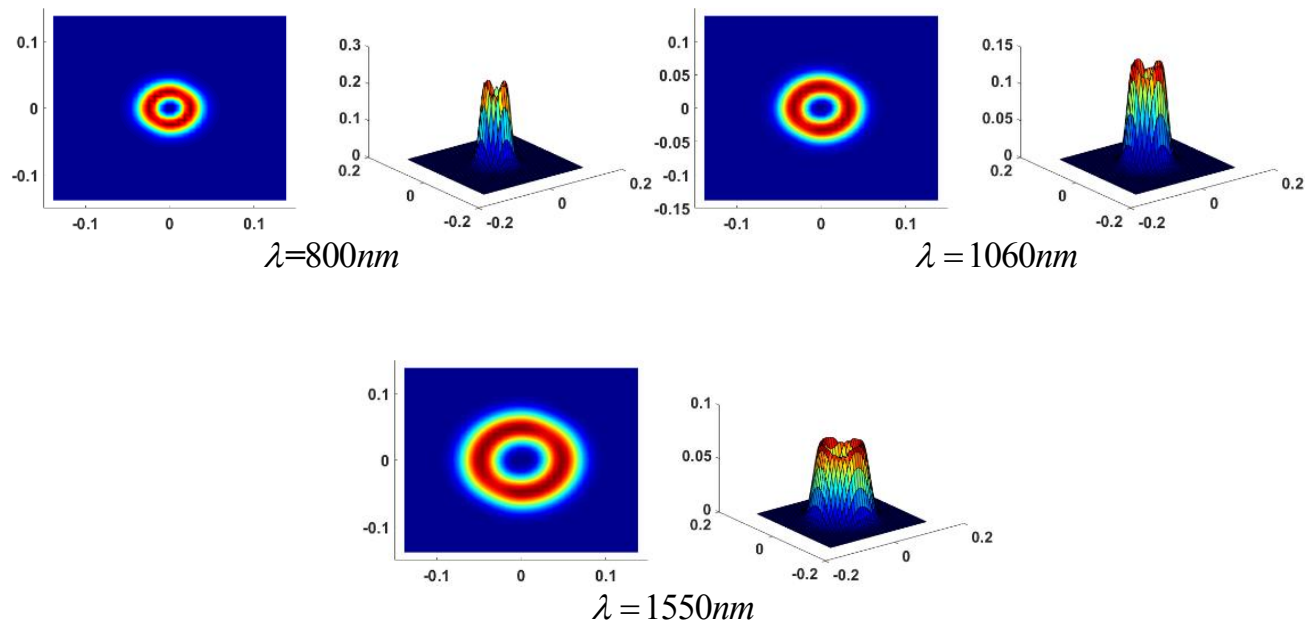

Figure 5. Ideal receiver intensity pictures of modified Bessel-Gaussian beams at various wave lengths.

Next ideal receiver intensity of modified Bessel-Gaussian beams at various wavelengths are examined. Fig 5 shows that as wavelength increases, the optical intensity was improved.

\subsubsection{Actual receiver intensity}

Now, the actual receiver intensity graphs that consider the scintillation shall be offered. Fig 6 exhibits actual receiver intensity pictures of modified Bessel-Gaussian beams at various wave lengths. From Fig 6, know that increase in wavelength will develop the intensity.

$$
a_{B}=200 \mathrm{~m}^{-1}, L=1 \mathrm{~km}, n=2, C_{n}^{2}=1 \times 10^{-15} \mathrm{~m}^{-2 / 3}
$$
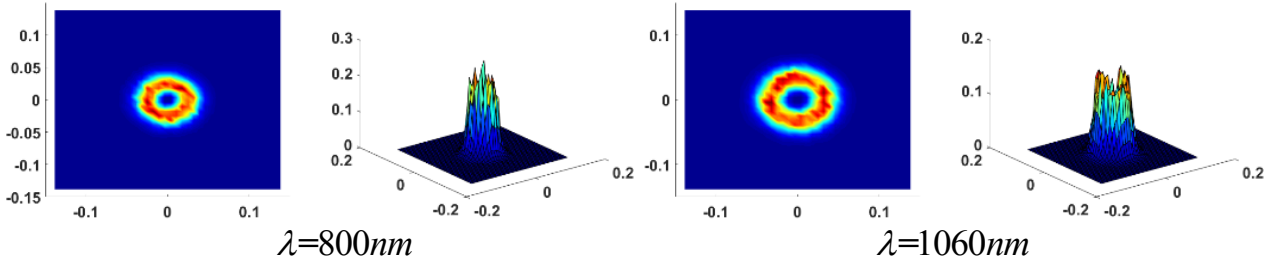


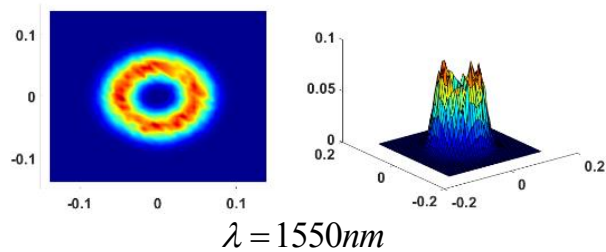

Figure 6. Actual receiver intensity pictures of modified Bessel-Gaussian beams at various wave lengths.

$$
\lambda=1550, a_{B}=200 m^{-1}, L=1 k m, n=2
$$
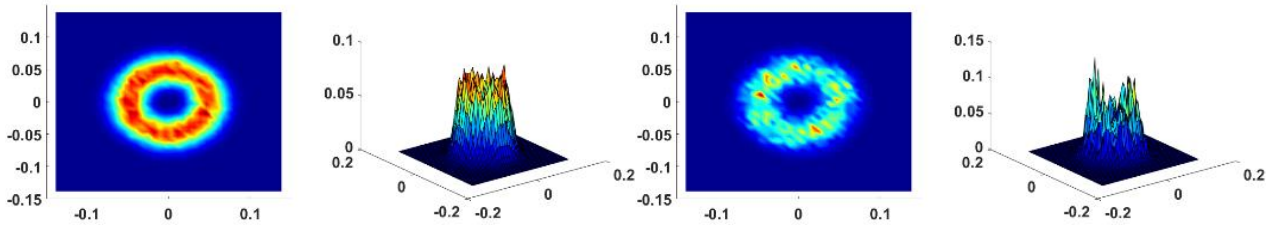

$$
C_{n}^{2}=1 \times 10^{-15} \mathrm{~m}^{-2 / 3}
$$

$C_{n}^{2}=1 \times 10^{-14} \mathrm{~m}^{-2 / 3}$
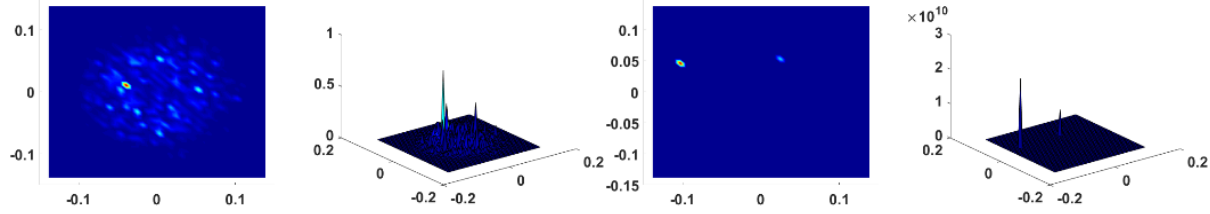

$C_{n}^{2}=1 \times 10^{-13} \mathrm{~m}^{-2 / 3}$

$C_{n}^{2}=1 \times 10^{-12} m^{-2 / 3}$

Figure 7. Actual receiver intensity pictures of modified Bessel-Gaussian beams at various $C_{n}^{2}$ values.

Fig 7 shows the actual receiver intensity of modified Bessel-Gaussian beams at various $C_{n}^{2}$ values. It is seen that, in the case of weak turbulence, the intensity distribution is very regular. Under the strong turbulence of $C_{n}^{2}=1 \times 10^{-12}$, the receiver intensity has lost its shape, and the optical beams become unrecognizable, which has caused FSO communication interruption.

$$
\lambda=1550, n=0, a_{B}=250 \mathrm{~m}^{-1}, C_{n}^{2}=1 \times 10^{-15} \mathrm{~m}^{-2 / 3}
$$
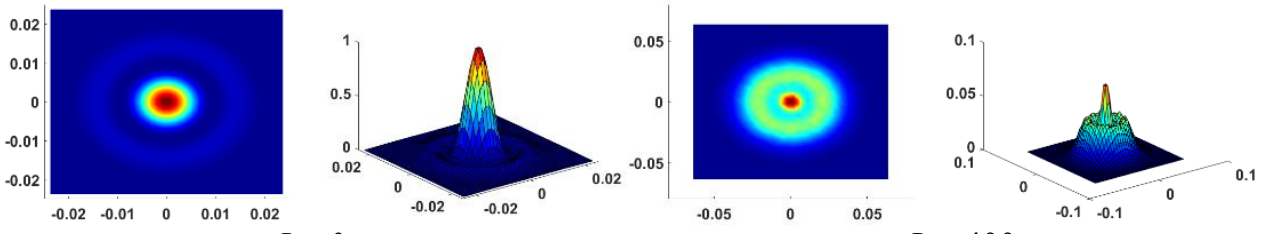

$L=0 m$

$L=400 m$
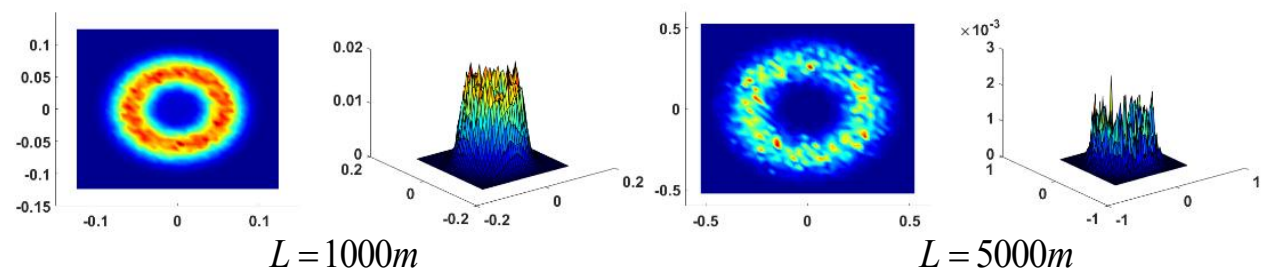

Figure 8. Actual receiver intensity of modified Bessel-Gaussian beams at various propagation lengths.

Fig 8 is the actual receiver intensity of modified Bessel-Gaussian beams at various propagation lengths. It shows that, the received beam is in a great shape and the intensity fluctuation is small when in a short propagation length. With the increase of transmission distance, the optical intensity becomes smaller and fluctuations increase. 


\subsection{LDPC code performance analysis}

When the modified Bessel-Gaussian beam is transmitted into the atmosphere, the presence of atmospheric turbulence would have an impact on the FSO system. Hence, we introduce the bit error rate(BER) performance of LDPC codes under turbulence in this section.

Fig 9 shows the effect of bit rate on performance of the QC-LDPC code. From Fig 9, we can know that with the increase of the code rate, the error rate of the QC-LDPC code constantly decreases, indicating that the performance of the QC-LDPC code becomes better and better.

Then we give out the influence of iteration times on performance of QC-LDPC codes. Fig 10 shows that increasing the number of decoding iterations, the performance of bit error rate reduction QC-LDPC code will be improved. However, when the number of iterations is large enough, only the delay and complexity of the system is increased as it increased, while the performance of the QC-LDPC code will no longer improve.

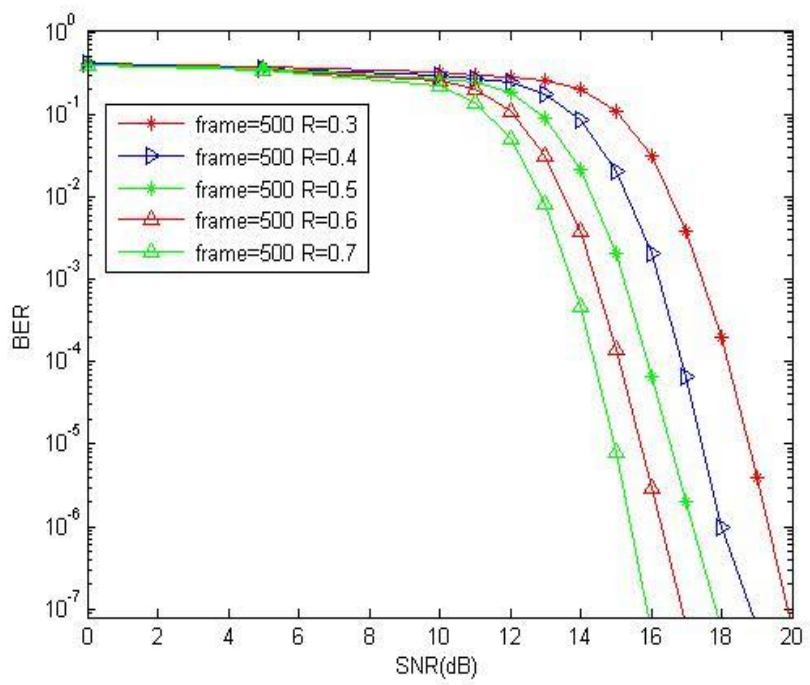

Figure 9. Effect of Bit Rate on Performance of QC-LDPC code

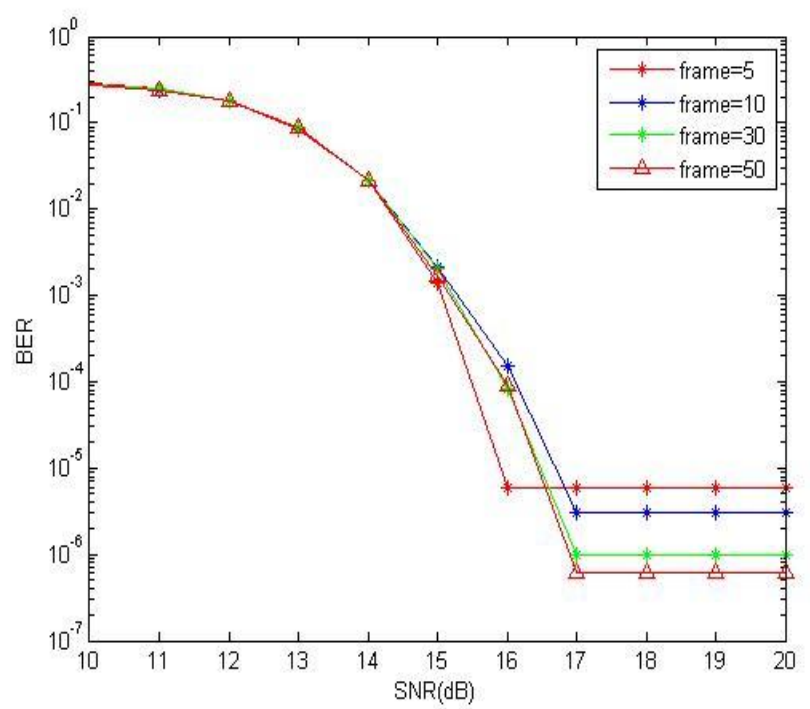

Figure 10. Influence of iteration times on performance of QC-LDPC. 


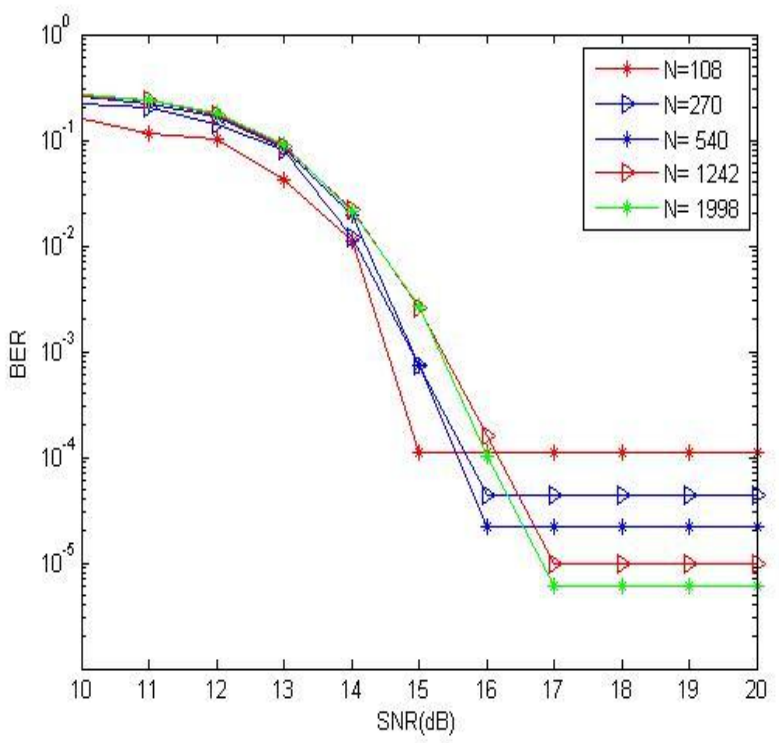

Figure 11. Influence of Code Length on Performance of QC-LDPC.

From the Fig 11, it is seen that the long code error performance of QC-LDPC code is better than that of short code. The code performance under a certain code length has a certain limit. As the code length increases, the complexity of encoding and decoding also increases, and the coding performance will be closer to the limit, and its performance will improve relatively as the code length increases.

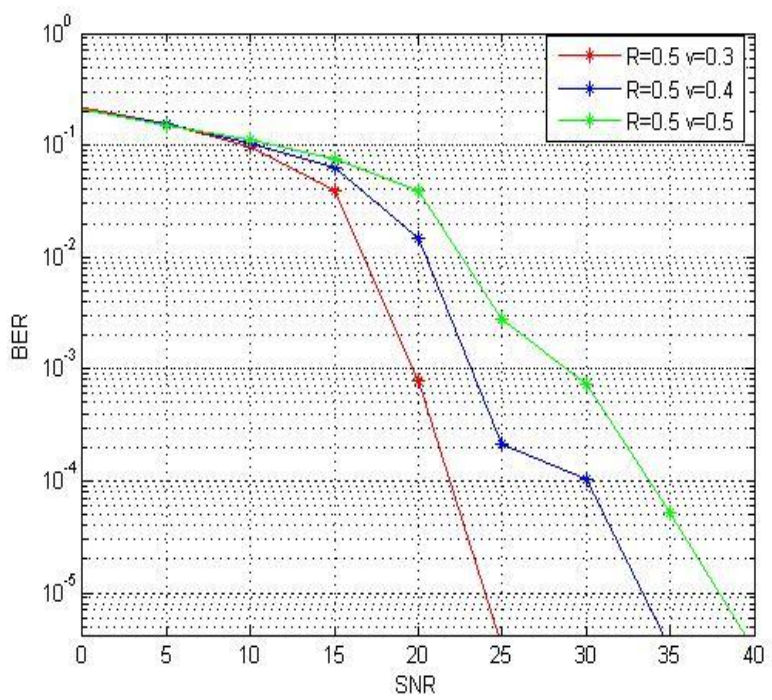

Figure 12. The Effect of Turbulent Intensity on the Performance of QC-LDPC Code under Weak Turbulence

Then we exhibit the effect of turbulent intensity on the performance of QC-LDPC Code under weak turbulence. Fig 12 shows that as the turbulence intensity increases, the bit error rate continues to increase and the performance of the QC-LDPC code becomes worse.

Now, the effect of turbulent intensity on the performance of QC-LDPC code under strong turbulence condition shall be offered. It is known that under certain conditions, the smaller the BER, the better the QC-LDPC code performance as the turbulence intensity decreases from the Fig 13. 


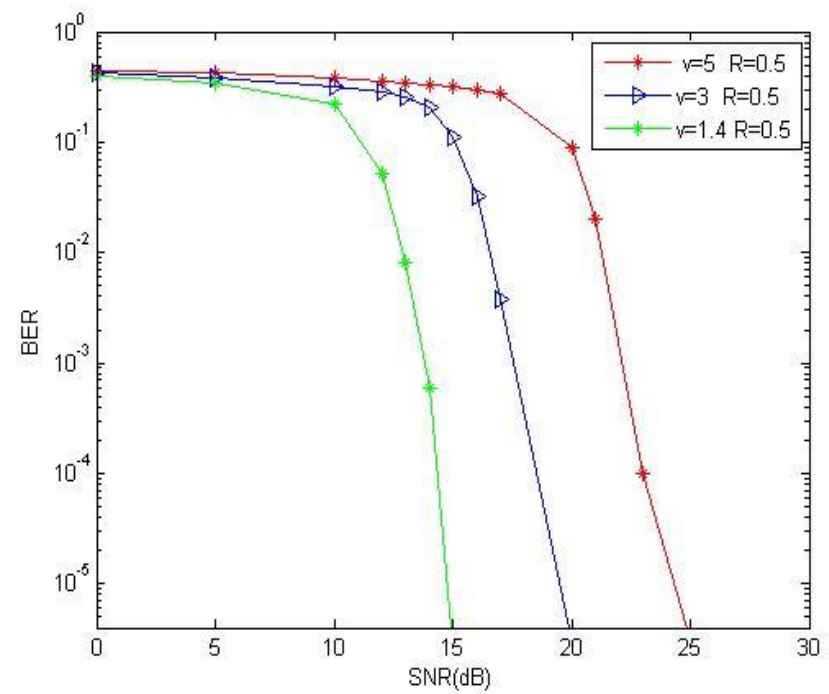

Figure 13. The Effect of Turbulent Intensity on the Performance of QC-LDPC Code under Strong Turbulence

\section{Conclusions}

For FSO communication system, we propose modified Bessel-Gaussian beams and LDPC codes to reduce the influence of atmospheric turbulence. We conclude that the order affects the Bessel part of the beam and changes the beam profile and outer ring, and that width parameter will affect the weights occupied by the Gaussian and Bessel parts of the beam. When the width increases, the beam will be biased towards the Bessel beam's shape and characteristics, whereas the width will be biased toward the Gaussian beam. In addition, increase in the transmission distance and refractive index structure constants will make the intensity of light scintillation more intense, both of which have an influence on the beam profile and shape. Under weak turbulence, as wavelength increases, it will improve the light intensity fluctuation. Finally, we find out that the interaction of the transmission distance, refractive index structure constant, and wavelength will affect the imaginary term in the beam, allowing the beam to exhibit the properties of the modified Bessel-Gauss beam. For the LDPC codes, we discover that under weak and strong turbulence conditions, the performance of the QC-LDPC code becomes better and better as the code rate increases, and the bit error rate keeps decreasing as the code rate increases.

\section{Reference}

1. H. Tian, L. Guo, P. Wang, et al. Optics Express, 25, 19995-20011 (2017).

2. K. Davaslioglu, E. ÇAğIral, M. Koca, Optics Express, 18, 16618-27(2010).

3. H.T. Eyyuboğlu, F. Hardalaç, Optics \& Laser Technology, 40, 343-351 (2008).

4. $\quad$ Myung S, Yang K, Kim J. IEEE Trans.inf.theory, 51, 2894-2901 (2005).

5. Y. Zhu, Int. J. Mod Phys B, 30, 1650193 (2016).

6. H.T. Eyyuboğlu, Appl. Phys. B, 88, 259-265 (2007).

7. L.C. Andrews, R.L. Phillips. Bellingham: SPIE Press, (1998).

8. D.J.C. MacKay, IEEE Trans. on Information Theory, 45, 399-431(1999).

9. Ivan B. Djordjevic, OPT. COMMUN. NETW, 2, (2010).

10. J. Wang, C.P. Udeh, Free-Space Laser Communication and Atmospheric Propagation XXIX. International Society for Optics and Photonics, 1009609. (2017). 